\title{
A transfer function approach to the small-signal response of saturated semiconductor optical amplifiers
}

Nielsen, Mads Lønstrup; Blumenthal, D. J.; Mørk, Jesper

Published in:

Journal of Lightwave Technology

Link to article, DOI:

$10.1109 / 50.908827$

Publication date:

2000

Document Version

Publisher's PDF, also known as Version of record

Link back to DTU Orbit

Citation (APA):

Nielsen, M. L., Blumenthal, D. J., \& Mørk, J. (2000). A transfer function approach to the small-signal response of saturated semiconductor optical amplifiers. Journal of Lightwave Technology, 18(12), 2151-2157.

https://doi.org/10.1109/50.908827

\section{General rights}

Copyright and moral rights for the publications made accessible in the public portal are retained by the authors and/or other copyright owners and it is a condition of accessing publications that users recognise and abide by the legal requirements associated with these rights.

- Users may download and print one copy of any publication from the public portal for the purpose of private study or research.

- You may not further distribute the material or use it for any profit-making activity or commercial gain

- You may freely distribute the URL identifying the publication in the public portal 


\title{
A Transfer Function Approach to the Small-Signal Response of Saturated Semiconductor Optical Amplifiers
}

\author{
Mads L. Nielsen, Daniel J. Blumenthal, Senior Member, IEEE, Member, OSA, and Jesper Mørk
}

\begin{abstract}
A theoretical analysis of the small-signal frequency response (SSFR) of a wavelength converter based on cross-gain modulation in a semiconductor optical amplifier with a finite waveguide loss is presented. We use a transfer function formalism to explain the resonant behavior of the frequency response. The limitations to the magnitude of the spectral overshoot are also accounted for. Operating with the data and CW signals in a co-propagating configuration, we find that the resonance only exists for a finite waveguide loss. In a counter-propagating scheme, a resonance can exist regardless of the presence of loss.
\end{abstract}

Index Terms-Cross-gain modulation, frequency response, overshoot, resonance, semiconductor optical amplifiers, wavelength conversion.

\section{INTRODUCTION}

$\mathbf{S}$ EMICONDUCTOR optical amplifier wavelength converters (SOA-WCs) have the potential to support wavelength switching in WDM transmission systems and optical networks [1]. WCs using cross-gain modulation (XGM) have been demonstrated at bit rates as high as $100 \mathrm{~Gb} / \mathrm{s}$ [2]. The operation of SOA-WCs under such wideband digital operation and high bit rates requires a detailed understanding of the WC frequency response. Several results on the modeling of small-signal frequency response (SSFR) of SOA-WCs based on XGM have been reported [3]-[7]. Mecozzi [3] developed an analytical expression for the SSFR taking into account the spatial inhomogeneity of the gain as well as the photon density. The analysis in [3] leads to the conclusion that the internal loss in the waveguide causes an overshoot in the SSFR at intermediate modulation frequencies, which has also been suggested by Mikkelsen [8] based on numerical simulations and measurements, as well as seen experimentally by Marcenac et al. [7]. Mørk et al. [9] have reported an analogous resonance in the SSFR of an SOA with a modulated injection current.

In this paper we use a transfer function formalism similar to that presented in [10], which explains the small-signal overshoot phenomena and aids in understanding limits to the overshoot. We are considering the situation where the SOA-WC based on $\mathrm{XGM}$ is operated with the data and CW signals in a co-propagating configuration, since the counter-propagation scheme suf-

Manuscript received January 4, 2000; revised September 11, 2000.

M. L. Nielsen and J. Mørk are with Research Center COM, Technical University of Denmark, DK-2800 Lyngby, Denmark.

D. J. Blumenthal is with the Optical Communications and Photonics Networks Laboratory, Department of Electrical and Computer Engineering, University of California, Santa Barbara, CA 93106 USA.

Publisher Item Identifier S 0733-8724(00)10543-2.

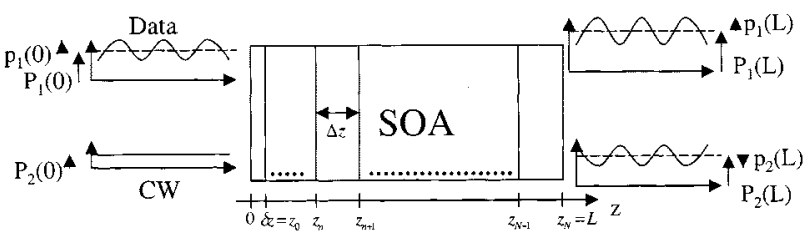

Fig. 1. SOA in a XGM wavelength conversion scheme. The discretization used in Section III is indicated.

fers from severe speed limitations [11]. However, counter-propagation is observed to lead to resonance even for the case of zero waveguide loss, and this will be explained and discussed. The paper is organized as follows: In Section II, the SSFR analysis presented in [3], which forms the basis for our investigation, will be briefly reviewed and the results cast in a suitable form. These results are used in Section III to define and analyze local transfer functions, which relate the small-signal modulation at the input and output of a small section of the amplifier. In Section IV the transfer function formalism is used to investigate the possible resonance of the XGM SSFR in the co-propagating configuration, with a brief discussion of the counter-propagation scheme. Finally, conclusions are made in Section V.

\section{THEORY}

Following Mecozzi [3], we consider an SOA in a XGM wavelength conversion scheme. This is shown in Fig. 1, where $P_{1}(z)$ and $P_{2}(z)$ are the average powers of the data channel and $\mathrm{CW}$ channel at the position $z$, respectively. The sinusoidal smallsignal modulation is of the form

$$
\begin{gathered}
P_{k}(z, t)=P_{k}(z)+p_{k}(z, \Omega) e^{j \Omega t}+p_{k}^{*}(z, \Omega) e^{-j \Omega t} \\
k=1,2
\end{gathered}
$$

where

$$
\begin{array}{ll}
P_{k}(z, t) & \text { total, instantaneous power in channel } k(k=1: \\
& \text { data ch., } k=2: \mathrm{CW} \mathrm{ch.}) ; \\
p_{k}(z, \Omega) & \text { modulated part of the power in channel } k ; \\
\Omega & \text { modulation angular frequency. }
\end{array}
$$

The SOA is characterized by the length $L$, the internal loss $\alpha_{\text {int }}$, the optical confinement factor $\Gamma$, the spontaneous carrier lifetime $\tau_{s}$, and the saturation power $P_{s a t}=A \hbar \omega /\left(a \Gamma \tau_{s}\right)$, where $A$ is the area of the active region, $\omega$ is the optical carrier frequency, and $a$ is the differential gain. The spontaneous recombination rate can be expressed as $R(N)=A N+B N^{2}+C N^{3}$ 
[12], where $A, B$, and $C$ are the nonradiative recombination coefficient due to traps and defects, the spontaneous radiative recombination coefficient, and the Auger coefficient, respectively. The assumption made in [3], that $\tau_{s}$ is independent of the carrier density variations inside the SOA, implies that $R(N)$ is approximated by $N / \tau_{s}$. In order for this approximation to be reasonably accurate, $\tau_{s}$ must be chosen as $\tau_{s}=\left[\left.(d R / d N)\right|_{\bar{N}}\right]^{-1}$, where $\bar{N}$ is an average of the carrier density over the length of the amplifier. This makes $\tau_{s}$ and consequently $P_{\text {sat }}$ independent of $z$. In addition, because $\omega$ and $a$ are assumed to be the same for both channels, $P_{\text {sat }}$ also becomes independent of wavelength. We ignore ASE, which restricts us to look at cases where the carrier depletion is dominated by the input signals.

Defining the sums $P_{T}(z)=P_{1}(z)+P_{2}(z)$ and $p_{T}=p_{1}(z)+$ $p_{2}(z)$, the small-signal modulation amplitudes can be shown to have the following spatial dependence [3]

$$
\begin{aligned}
p_{k}(z, \Omega)=G(z) \times\{ & p_{k}(0, \Omega)-\frac{P_{k}(0) p_{T}(0, \Omega)}{P_{T}(0)} \\
& \cdot(1-\exp [-K(z, \Omega)]\}
\end{aligned}
$$

where $p_{T}(0)=p_{1}(0)$ because the $\mathrm{CW}$ channel, per definition, lacks modulation at the input of the SOA. $K(z, \Omega)$ is given by [3]

$$
\begin{aligned}
& K(z, \Omega) \frac{1}{1-j \Omega \tau_{s} \xi} \\
& \quad \times\left\{\xi \ln \frac{G_{0}(z)}{G(z)}-\ln \left[1-\frac{\frac{(G(z)-1) P_{T}(0)}{P_{\text {sat }}}}{1+\frac{G(z) P_{T}(0)}{P_{\text {sat }}}-j \Omega \tau_{s}}\right]\right\} .
\end{aligned}
$$

In (3), $\xi=\alpha_{\text {int }} /\left(\Gamma g_{0}\right)$ is the normalized internal loss, where $g_{0}$ is the unsaturated material gain coefficient. $G_{0}(z)=\exp \left[\Gamma g_{0}(1-\xi) z\right]$ is the unsaturated amplifier gain, measured between the input facet and the spatial coordinate $z$, and $G(z)=P_{T}(z) / P_{T}(0)$ is the corresponding saturated gain that can be found from $G_{0}(z)$ and $P_{T}(0)$ [3].

In the limit $z \rightarrow 0^{+}$, the relative SSFR for the CW channel $p_{2}(z, \Omega) / p_{2}(z, 0)$ turns out to be a simple low-pass characteristic. This is not easily realized because of the complexity of (2) and (3), but by returning to the differential equation that (2) is the solution of [3], [6]

$$
\frac{d p_{k}}{d z}=\left(g-\alpha_{i n t}\right) p_{k}-\frac{g P_{k}(z) / P_{s a t}}{1+P_{T}(z) / P_{s a t}-j \Omega \tau_{s}}\left(p_{1}(z)+p_{2}(z)\right)
$$

where $g=\Gamma g_{0} /\left(1+P_{T} / P_{\text {sat }}\right)$ is the saturated gain coefficient, we are able to derive an expression for the low-pass function. By linearizing (4) for $k=2$

$$
\begin{aligned}
p_{2}(z, \Omega) & \approx p_{2}(0, \Omega)+\left.\frac{d p_{2}(z, \Omega)}{d z}\right|_{z=0}(z-0) \\
& =-\frac{g P_{2}(0) / P_{\text {sat }}}{1+P_{T}(0) / P_{\text {sat }}-j \Omega \tau_{s}} p_{1}(0) z
\end{aligned}
$$

the relative SSFR $L(\Omega)$ of the CW channel after the input facet can be expressed

$$
L(\Omega)=\lim _{z \rightarrow 0^{+}} \frac{p_{2}(z, \Omega)}{p_{2}(z, 0)}=\frac{\left.\frac{d p_{2}(z, \Omega)}{d z}\right|_{z=0}}{\left.\frac{d p_{2}(z, 0)}{d z}\right|_{z=0}}=\frac{1}{1-j \Omega \tau_{\mathrm{eff}}(0)}
$$

where $\tau_{\text {eff }}(z)$ is the effective carrier lifetime given by

$$
\tau_{\mathrm{eff}}(z)=\frac{\tau_{s} \tau_{s t}(z)}{\tau_{s}+\tau_{s t}(z)}=\frac{P_{s a t}}{P_{s a t}+G(z) P_{T}(0)} \tau_{s}
$$

and $\tau_{s t}=P_{\text {sat }} \tau_{s} /\left(G(z) P_{T}(0)\right)$ is the stimulated contribution to the carrier lifetime. $L(\Omega)$ is a simple low-pass function with a high-frequency slope of $-10 \mathrm{~dB} / \mathrm{dec}$ ade, and a corner frequency determined solely by the effective carrier lifetime $\tau_{\text {eff }}(0)$ at the input facet. It is important to stress that the expression given in (6) is exact, since the linearization made in (5) becomes exact in the limit $z \rightarrow 0^{+}$, which will be referred to as $z=0^{+}$ from now on. A similar analysis for the data channel shows that $p_{1}(z, \Omega) / p_{1}(z, \Omega)$ is a high pass function which flattens out in the high- and low frequency limits, that is, it has an equal number of poles and zeroes. This is the case for any $z$.

\section{LOCAL TRANSFER FUNCTIONS}

Although the equations presented above are continuous in the sense that $z$ can take on any value between 0 and $L$, we will now divide the SOA into $M$ sections (see Fig. 1), all of length $\Delta z=L / M$. The discretization enables us to define local transfer functions $T_{k}\left(z_{n}, \Omega\right), k=1,2$, connecting the input and output of a section located between $z_{n}=n \Delta z$ and $z_{n+1}$

$$
T_{k}\left(z_{n}, \Omega\right)=\frac{p_{k}\left(z_{n+1}, \Omega\right)}{p_{k}\left(z_{n}, \Omega\right)}, \quad n=0,1,2 \cdots M-1 .
$$

The first section $(n=0)$ starts at $z=z_{0}=0^{+}$and ends at $z=z_{1}=\Delta z$, that is, it does not include the input facet. This definition of the first section ensures that $p_{2}\left(z_{0}, \Omega\right)$ is finite for $p_{1}(0)>0$, and consequently that the definition of $T_{2}\left(z_{0}, \Omega\right)$ is valid. The transfer function approach is practical since there is only modulation on one of the input channels (the data channel), which makes it possible to relate an arbitrary $p_{k}\left(z_{n}, \Omega\right)$ to $p_{1}(0, \Omega)$. In the general case, with modulation on both signals at the input, a transfer matrix approach is more appropriate. Using (8) successively, the relative SSFR of the CW channel at the output of the amplifier can be expressed

$$
\frac{p_{2}(L, \Omega)}{p_{2}(L, 0)}=\Pi_{2}(\Omega) L(\Omega)
$$

where

$$
\Pi_{2}(\Omega)=H\left(z_{M-1}, \Omega\right) H\left(z_{M-2}, \Omega\right) \cdots H\left(z_{0}, \Omega\right)
$$

and

$$
H\left(z_{n}, \Omega\right)=\left(\frac{T_{2}\left(z_{n}, \Omega\right)}{T_{2}\left(z_{n}, 0\right)}\right) .
$$




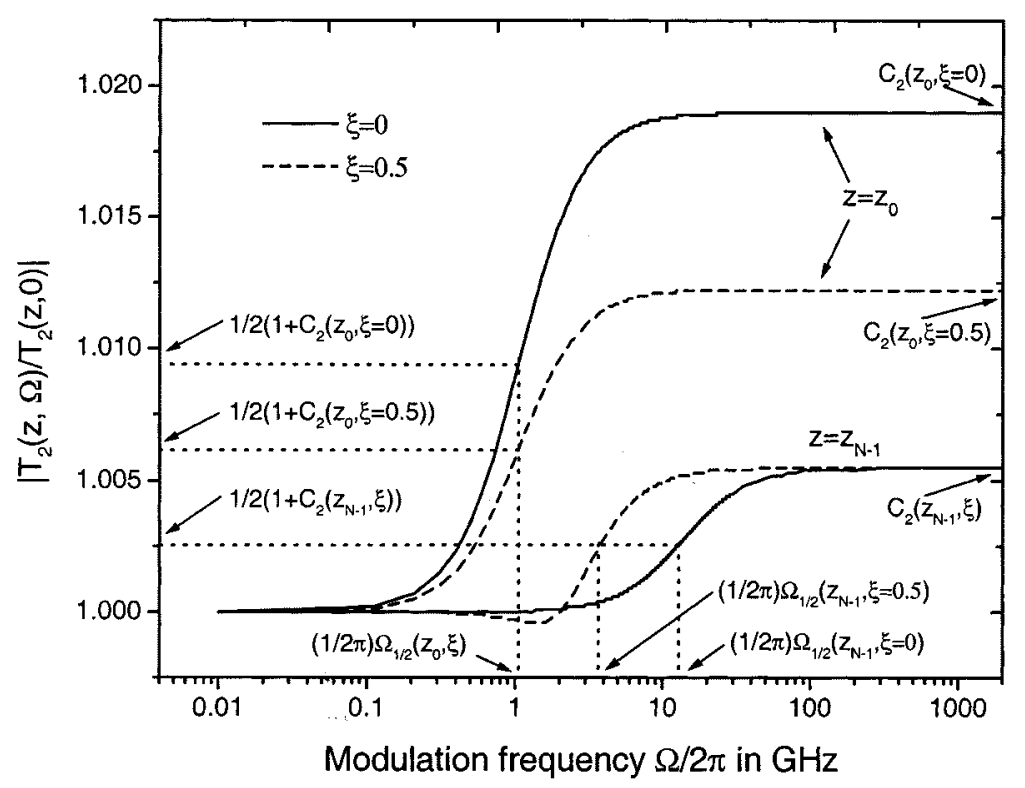

Fig. 2. Examples of $\mathrm{CW}$-transfer functions. The spectral position $\Omega_{1 / 2}$ is defined as the angular frequency where the transfer function is halfway between the low- and high-frequency limits. The spectral drift is seen to be more pronounced for $\xi=0$ than for $\xi=0.5$.

$H\left(z_{n}, \Omega\right)$ are the individual transfer functions normalized to the low-frequency response. Using (2), (3), (7), and (11), the relative transfer functions can be expressed as

$$
\begin{aligned}
& H\left(z_{n}, \Omega\right) \\
& \quad=\frac{G\left(z_{n}\right)-1}{G\left(z_{n+1}\right)-1} \frac{\tau_{\mathrm{eff}}\left(z_{n}\right)}{\tau_{\mathrm{eff}}\left(z_{n+1}\right)} \frac{1-\exp \left[-K\left(z_{n+1}, \Omega\right)\right]}{1-\exp \left[-K\left(z_{n}, \Omega\right)\right]} .
\end{aligned}
$$

Making use of L'Hospital's rule, it can be shown that the highfrequency limit of (12) is given by

$$
\begin{aligned}
\lim _{\Omega \rightarrow \infty} & H\left(z_{n}, \Omega\right) \\
= & \frac{G\left(z_{n}\right)-1}{G\left(z_{n+1}\right)-1} \frac{\tau_{\text {eff }}\left(z_{n}\right)}{\tau_{\text {eff }}\left(z_{n+1}\right)} \\
& \times \frac{\ln \left(1-\xi \tau_{s} / \tau_{\text {eff }}(0)\right)-\ln \left(1-\xi \tau_{s} / \tau_{\text {eff }}\left(z_{n+1}\right)\right)}{\ln \left(1-\xi \tau_{s} / \tau_{\text {eff }}(0)\right)-\ln \left(1-\xi \tau_{s} / \tau_{\text {eff }}\left(z_{n}\right)\right)} .
\end{aligned}
$$

Since $G\left(z_{n+1}\right)>G\left(z_{n}\right)$ and $\tau_{\text {eff }}\left(z_{n+1}\right)<\tau_{\text {eff }}\left(z_{n}\right)$ as long as $g>\alpha_{i n t}$, which is the regime we are operating in, it can be shown that $\left|\lim _{\Omega \rightarrow \infty} H\left(z_{n}, \Omega\right)\right|>1$. This means that $H\left(z_{n}, \Omega\right)$ is a high-pass function with an equal number of poles and zeroes. Notice that this does not rule out the existence of local extremes at intermediate modulation frequencies (see Fig. 2). It is important to realize, that the high-pass response of an individual section only makes sense because the section is inside the amplifier. If the section was alone, corresponding to a short SOA of one section length $L / M$, it would obviously have a low-pass characteristic determined by its own input facet. We define the contrast ratio $C_{2}\left(z_{n}\right)$ of the transfer functions as the ratio of the function in the high- and low-frequency limits

$$
C_{2}\left(z_{n}\right)=\left|\lim _{\Omega \rightarrow \infty} H\left(z_{n}, \Omega\right)\right| .
$$

Returning to (9), it becomes apparent that the relative SSFR of the $\mathrm{CW}$ channel at the output of the SOA is the product of the simple low-pass function $L(\Omega)$ and a series of high-pass functions $H\left(z_{n}, \Omega\right)$ with equal numbers of poles and zeroes. The high-pass functions will tend to compensate the low-pass function, and consequently increase the bandwidth of the XGM response. This is equivalent to what Durhuus et al. [5] refer to as "the saturation filtering effect." Examples of the high-pass functions are shown in Fig. 2 for $z=z_{0}$ and $z=z_{M-1}$ for the normalized losses $\xi=0$ and $\xi=0.5$. The definitions of Fig. 2 will be explained in the next section, where a list of parameters can also be found.

\section{The ResonAnCE PEAK}

We will now use formalism introduced in the previous section to explain why the SSFR of the CW channel may display a resonance when loss is introduced in the waveguide. First, we will define the spectral position $\Omega_{1 / 2}$ of a relative transfer function $H\left(z_{n}, \Omega\right)$ as the frequency at which it has reached the point halfway in between the low- and high-frequency limits. The definition is shown graphically in Fig. 2. Other definitions have been considered, but since they all gave rise to the same conclusions, we chose this one because of its simplicity. In Figs. 2-7, the following set of parameters are used: $P_{T}(0)=3 \mathrm{~mW}, P_{\text {sat }}=10 \mathrm{~mW}, \tau_{s}=200 \mathrm{ps}, \Gamma=0.4$, $g_{0}=5 \cdot 10^{4} \mathrm{~m}^{-1}$, and $M=200$. The magnitude of $M$ does not change the total SSFR of the SOA. However, the characteristics of the individual transfer functions will obviously change with $M$, since the product of all of them is independent of $M$. The amplifier length $L$ is $1 \mathrm{~mm}$ unless stated otherwise and the normalized loss $\xi$ will be varied.

It is observed in Fig. 2 that $\Omega_{1 / 2}$ is independent of $\xi$ for $z=$ $z_{0}$, which is anticipated since the loss has not yet had a chance to change the gain $G$ at this early stage of the SOA. The contrast ratio $C_{2}(z)$ is observed to be independent of $\xi$ in the rear of the SOA $\left(z=z_{N-1}\right)$. This is also verified in Fig. 6, and will 


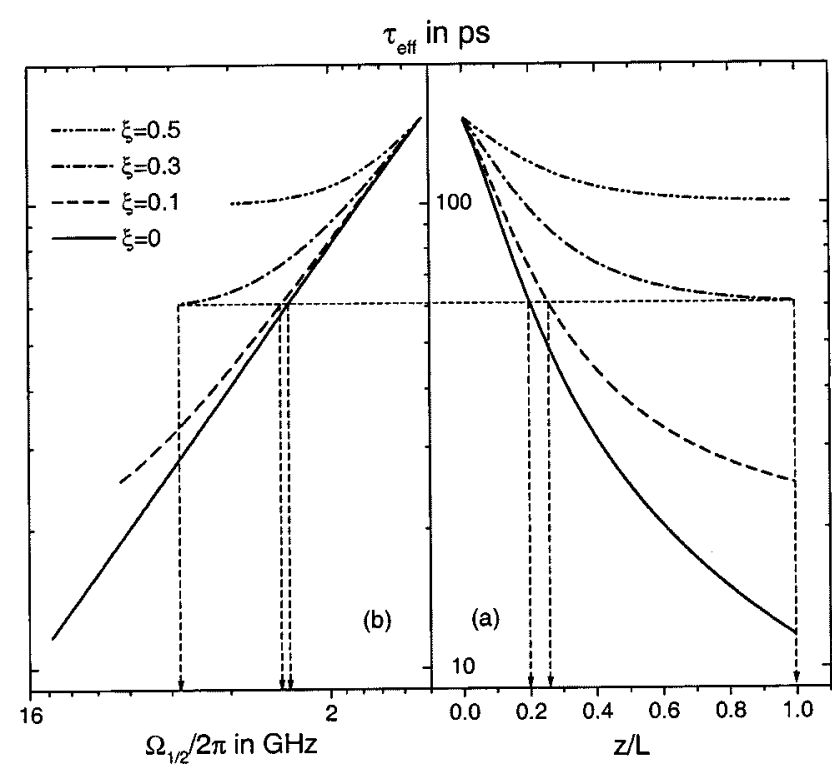

Fig. 3. (a) Effective carrier lifetime versus the normalized position inside the SOA for different values of the normalized loss. An increase of the loss causes an increase of the lifetime. (b) Spectral position versus the effective carrier lifetime. The vertical lines show that a specific lifetime will be reached later in the SOA when the loss is increased.

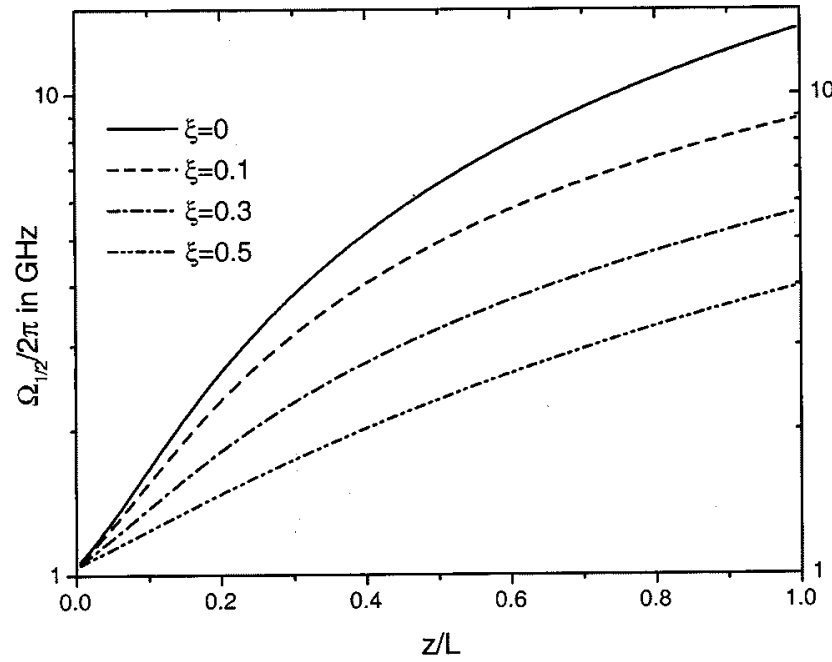

Fig. 4. Spectral position of CW-transfer functions as a function of the normalized position for different normalized losses. An increase of the loss is seen to inhibit the "drift" of the transfer functions.

be explained later. Finally, it is clear that the transfer functions "drift" less when loss is present, compared to the loss-less case. It will become clear in the following, that this is a very important feature of the $\mathrm{CW}$ transfer functions. At the rear of the amplifier, the gain in the lossy case is significantly smaller, which causes the effective carrier lifetime $\tau_{\text {eff }}(z)$ to increase, compared to the loss-loss case. This increase of $\tau_{\text {eff }}(z)$ with $\xi$ for constant $z$ is illustrated in Fig. 3(a), where $\tau_{\text {eff }}(z)$ is shown on a logarithmic scale as a function of the normalized position $z / L$. Since $\tau_{\mathrm{eff}}(z)$ is a decreasing function of $z$, and $\tau_{\text {eff }}(0)$ is independent of $\xi$, it follows that the average slope magnitude of $\tau_{\text {eff }}(z)$ will decrease as $\xi$ is increased. Fig. 3(b) shows $\Omega_{1 / 2}$ on a logarithmic scale as

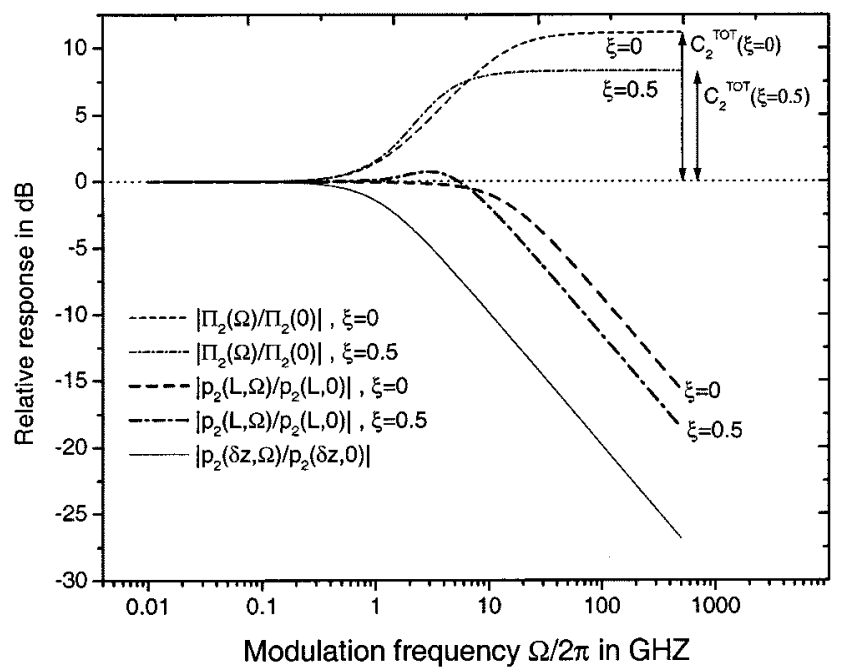

Fig. 5. Low-pass characteristic $L(\Omega)$, the total high-pass function $\Pi_{2}(\Omega)$, and the total, relative response, equal to the product of the two former (or the sum in this logarithmic chart), as a function of the modulation frequency for $\xi=0$ and $\xi=0.5$. The loss is seen to cause an overshoot and a reduction of the $3 \mathrm{~dB}$ bandwidth. The latter is due to the indicated reduction of the contrast ratio.

a function of $\tau_{\text {eff }}$, also on a logarithmic scale, for the same losses as in Fig. 3(a). Notice that the curve corresponding to $\xi=0$ is linear with a slope magnitude of 1 , indicating that $\Omega_{1 / 2}=$ $c \tau_{\text {eff }}^{-1}(z)$ for this case, where $c$ is a constant. As $\xi$ is increased, the curves deviate more and more from linearity, causing $\Omega_{1 / 2}$ to increase with $\xi$ for a constant $\tau_{\text {eff }}$. However, as illustrated in Fig. 3(a), a specific $\tau_{\text {eff }}$ is reached at a later stage in the amplifier when the loss is increased, due to the reduced change of $\tau_{\text {eff }}$ over the length of the SOA.

Fig. 4 shows the evolution of $\Omega_{1 / 2}$ with $z / L$, and here it is clear that the "drift" of the transfer functions is reduced significantly when $\xi$ is increased. That means that in the cases where loss is present, the high-pass functions $H\left(z_{n}, \Omega\right)$ will tend to align themselves. When they are multiplied according to (10) the alignment will cause the slope magnitude of the total high-pass function $\Pi_{2}(\Omega)$ to increase through resonant enhancement. The trend in Fig. 4 is clear: the bigger the loss, the more effectively the transfer functions are aligned. However, the alignment will only establish itself as an overshoot if a value of $\Omega$ exists, such that the logarithm of $\left|\Pi_{2}(\Omega)\right|$ has a larger slope magnitude than the logarithm of the low-pass function $|L(\Omega)|$. This can be shown in the following way:

$$
\begin{gathered}
\frac{d}{d \Omega}\left|\Pi_{2}(\Omega) L(\Omega)\right|>0 \Longrightarrow \frac{d}{d \Omega}\left(\log |L(\Omega)|+\log \left|\Pi_{2}(\Omega)\right|\right)>0 \\
\Longrightarrow \frac{d}{d \Omega} \log \left|\Pi_{2}(\Omega)\right|>\left|\frac{d}{d \Omega} \log \right| L(\Omega)||
\end{gathered}
$$

As long as (15) is satisfied, the response will increase with frequency. Whether this will be the case or not for a specific loss $\xi$, will depend on the amplifier length $L$. This will be discussed in more detail later.

Fig. 5 shows the low-pass function $L(\Omega)$, and the total high-pass function $\Pi_{2}(\Omega)$ for $\xi=0$ and $\xi=0.5$, as well as the relative SSFR of the CW channel at the output of the SOA. 


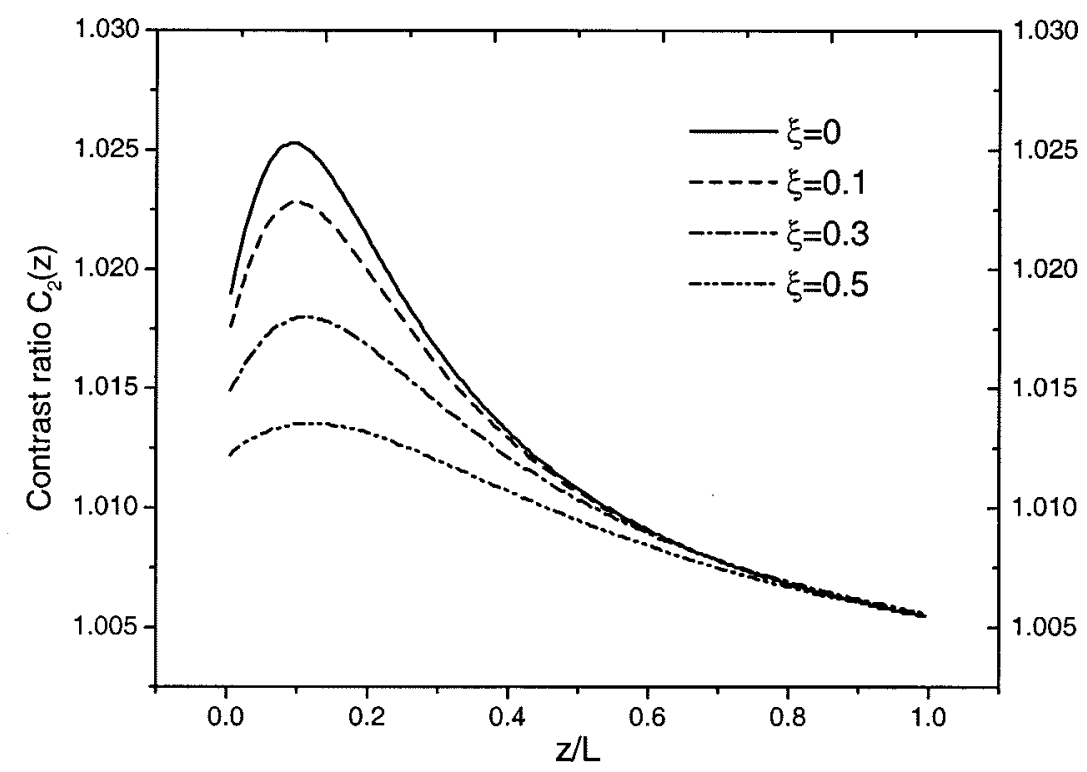

Fig. 6. Contrast ratio versus the normalized position. Increasing the loss decreases the contrast ratio. The maxima are due to the existence of inflection points in Fig. 3(a).

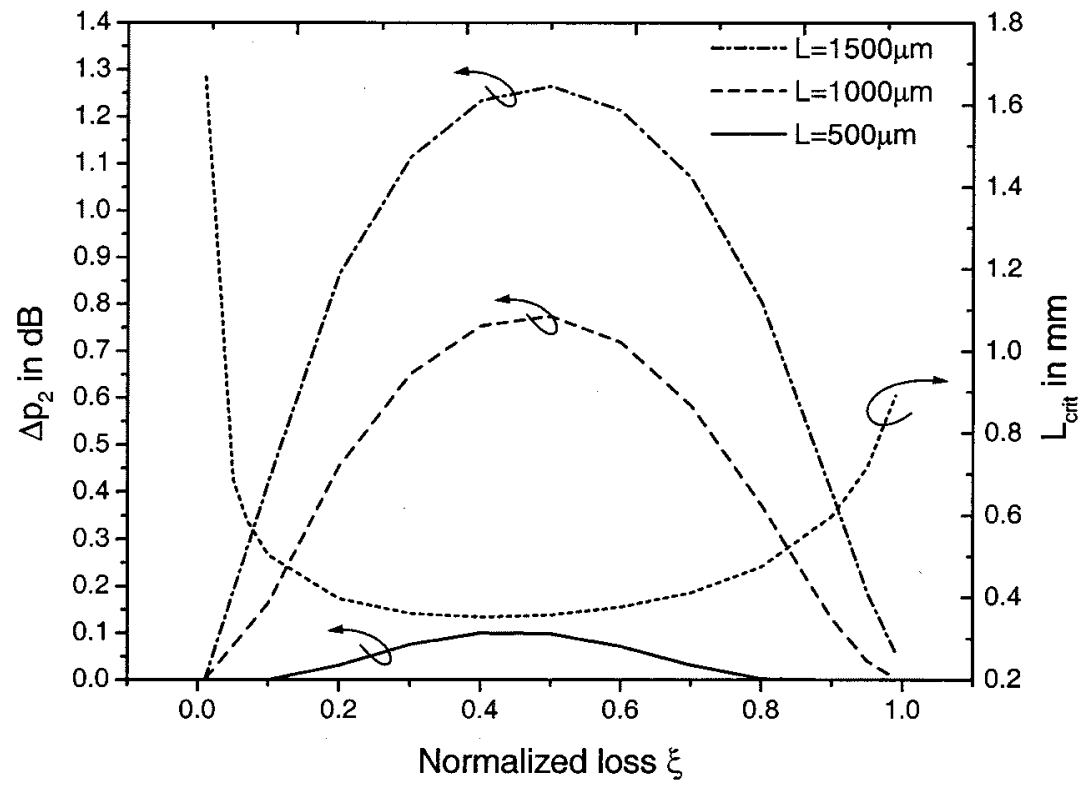

Fig. 7. Magnitude of the overshoot in decibels for three different amplifier lengths (left) and the critical length, below which no overshoot is observed (right), versus normalized loss. The effects of alignment and reduction of contrast ratio are in balance for $\xi=0.4-0.5$, depending slightly on the amplifier length. As the loss approaches zero, the critical length goes to infinity.

According to (9), the latter is the product of the two former, or in this logarithmic chart, the sum. It is clear from Fig. 5 that the higher slope of $\log \left|\Pi_{2}(\Omega)\right|$, in the lossy case, results in an overshoot when the low-pass function is added.

Neglecting the loss dependence of the contrast ratio $C_{2}\left(z_{n}\right)$, one would think that the magnitude of a possible overshoot would keep on increasing with $\xi$. However, the contrast ratio (14) reveals a significant $\xi$-dependence. This is shown in Fig. 6, where $C_{2}\left(z_{n}\right)$ is seen to decrease as $\xi$ is increased. This can be attributed to the reduction of the slope magnitude of $\tau_{\text {eff }}(z)$ in Fig. 3(a). The local maxima of the contrast ratio observed in Fig. 6 are due to the fact that the $\tau_{\text {eff }}$ versus $z$ curves in Fig. 3(a) have inflection points. As observed in Fig. 2 as well, the contrast ratio becomes independent of $\xi$ toward the end of the amplifier. This is because the slopes of the curves in Fig. 3(a) all approach the same value (zero) for $z / L$ approaching 1 .

The contrast ratio $\mathrm{C}_{2}^{T O T}$ of the total high-pass function $\Pi_{2}(\Omega)$ shown in Fig. 5, is the product of the contrast ratios $C_{2}\left(z_{n}, \Omega\right)$ of all the individual relative transfer functions $H\left(z_{n}, \Omega\right)$, as seen in (10). From Fig. 6 it is obvious that $C_{2}^{T O T}$ must decrease with $\xi$, and this is also clearly observed in Fig. 5. This is the reason why the $3 \mathrm{~dB}$ modulation bandwidth decreases when loss is introduced. The degradation of the contrast ratio reduces the slope of $\Pi_{2}(\Omega)$ which will decrease the 
overshoot, as discussed earlier. This means that the reduction of the contrast ratio and the alignment of the transfer functions have opposite effects on the magnitude of the overshoot. The former will reduce it, whereas the latter will tend to increase it. This is illustrated in Fig. 7 (left), where the magnitude of the overshoot $\Delta p_{2}$ is shown as a function of the normalized loss $\xi$ for three different amplifier lengths. The unsaturated gain coefficient $g_{0}$ is kept constant, corresponding to a constant injection current density. For the set of parameters chosen, it is observed that for normalized losses up to $\xi=0.4-0.5$, depending on the amplifier length, the alignment effect is dominant. At higher values of $\xi$ the degradation of the contrast ratio takes over. Fig. 7 (right) shows that for any given $\xi$ there is a critical amplifier length $L_{c r i t}$, below which no overshoot is observed. This effect has also been reported in the case where the bias current is modulated [9]. For $\xi$ approaching $0, L_{\text {crit }}$ goes to infinity, which is in agreement with the fact that no overshoot exists for loss-less waveguides operated in the co-propagating scheme [3], [4]. The critical length has a minimum around $\xi=0.4$. It is important to stress that $\xi$ can be manipulated by changing the bias current since $\xi$ depends on $g_{0}$.

If the data and $\mathrm{CW}$ channels are injected from opposite facets of the SOA, data at $z=0$ and $\mathrm{CW}$ at $z=L$, several things will change. First of all, the optical power may experience a local minimum inside the SOA, in which case the effective carrier lifetime $\tau_{\text {eff }}(z)$ will have a local maximum. This will have a strong impact on the transfer functions, as seen in (11)-(13). The low-pass function $L(\Omega)$ will also change, since its corner frequency will now be determined by $\tau_{\text {eff }}(L)$ at the output facet, as opposed to $\tau_{\text {eff }}(0)$ in the co-propagating scheme. Since the two can differ by an order of magnitude, see Fig. 3, the $3 \mathrm{~dB}$ bandwidth of (6) will change accordingly. Considering the special case where the average power in the $\mathrm{CW}$ channel is negligible compared to that of the data channel, $\tau_{\text {eff }}(z)$ will be independent of the propagation direction of the $\mathrm{CW}$ channel. However, the significant decrease of $\tau_{\text {eff }}(z)$ from the input- to the output facet (with respect to the data channel), pushes the corner frequency of (6) toward higher frequencies in the counter-propagating scheme, leaving the total high-pass function $\Pi_{2}(\Omega)$ unchanged. From Fig. 5 and (15) it is obvious that the total response will show a resonance in this case, because $L(\Omega)$ will have a negligible slope in a frequency span where the slope magnitude of $\Pi_{2}(\Omega)$ is finite. An important conclusion is, that the overshoot will exist regardless of the magnitude of the loss $\xi$, unlike in the co-propagating scheme.

It follows from the above that the modulation bandwidth of the SOA increases when operated in the counter-propagating configuration. This is not true in reality, however, because of walk-off effects [4], [11], which cause a steep fall-off of the SSFR when the modulation frequency becomes comparable to the transit time of the SOA [4]. However, the walk-off effects do not change the fact that the SSFR can display a resonance for zero waveguide loss. The predictions made above, based on the transfer function approach presented here, have been verified by an iterative small-signal model of the counter-propagation configuration.

\section{CONCLUSION}

A transfer function approach to analyzing the small-signal frequency response of the $\mathrm{CW}$ channel in a XGM wavelength conversion scheme has been presented. It is found that the existence of the resonance peak can be explained in terms of aligning high-pass transfer functions. The degradation of the response function that follows from the internal loss in the waveguide is also successfully explained from the transfer functions as a decrease of the contrast ratio. We find that no overshoot will be observed in the co-propagation scheme if the amplifier length is below a certain critical value. For the parameters used, we found that the critical length is minimum for the value $\xi=0.40$ of the normalized loss. Using the transfer function approach presented here, we conclude that a resonance of the SSFR can exist in the counter-propagating configuration, regardless of the presence of loss.

\section{REFERENCES}

[1] S. L. Danielsen, P. B. Hansen, and K. E. Stubkjær, "Wavelength conversion in optical packet switching," J. Lightwave Technol., vol. 16, pp. 2095-2108, 1998.

[2] A. D. Ellis, A. E. Kelly, D. Nesset, D. Pitcher, D. G. Moodie, and R. Kashyap, "Error free $100 \mathrm{gbit} / \mathrm{s}$ wavelength conveersion using grating assisted cross-gain modulation in $2 \mathrm{~mm}$ long semiconductor amplifiers," IEE Electron. Lett., vol. 34, pp. 1958-1959, 1998.

[3] A. Mecozzi, "Small-signal theory of wavelength converters based on cross-gain modulation in semiconductor optical amplifiers," IEEE Photon. Technol. Lett., vol. 8, pp. 1471-1473, 1996.

[4] D. Marcenac and A. Mecozzi, "Switches and frequency converters based on cross-gain modulation in semiconductor optical amplifiers," IEEE Photon. Technol. Lett., vol. 9, pp. 749-751, 1997.

[5] T. Durhuus, B. Mikkelsen, C. Jørgensen, S. L. Danielsen, and K. E. Stubkjær, "All-optical wavelength conversion by semiconductor optical amplifiers," J. Lightwave Technol., vol. 14, pp. 942-954, 1996.

[6] D. A. O. Davies, "Small-signal analysis of wavelength conversion in semiconductor laser amplifiers via gain saturation," IEEE Photon. Technol. Lett., vol. 7, pp. 617-619, 1995.

[7] D. D. Marcenac, A. E. Kelly, D. Nesset, and D. G. Moodie, "Speed and travelling wave effects in cross-gain modulation in semiconductor optical amplifiers," in Proc. COST239/240 Workshop Semiconductor Optical Amplifiers, Prague, Oct. 27-28, 1997.

[8] B. Mikkelsen, Ph.D. dissertation, Department of Electromagnetic Systems, Technical University of Denmark, 1994.

[9] J. Mørk, A. Mecozzi, and G. Eisenstein, "The modulation response of a semiconductor laser amplifier," IEEE J. Select. Topics Quantum Electron., vol. 5, pp. 851-860, 1999.

[10] A. Mecozzi and D. Marcenac, "Theory of optical amplifier chains," $J$. Lightwave Technol., vol. 16, pp. 745-756, 1998.

[11] D. Nesset, T. Kelly, and D. Marcenac, "All-optical wavelength conversion using soa nonlinearities," IEEE Commun. Mag., vol. 36, pp. 56-61, 1998.

[12] L. A. Coldren and S. W. Corzine, Diode Lasers And Photonic Integrated Circuits. New York: Wiley, 1995.

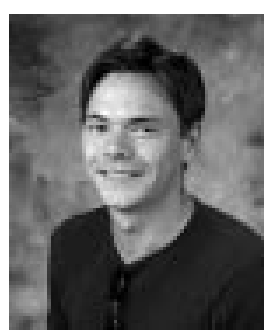

Mads L. Nielsen is in the final stage of the M.Sc. degree in applied physics at the Technical University of Denmark (TUD), Lyngby.

His main interests include wavelength conversion and switching in semiconductor optical amplifiers, with the emphasis on numerical modeling. 


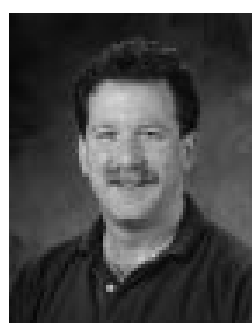

Daniel J. Blumenthal (S'91-M'93-SM'97) received the B.S.E.E. degree from the University of Rochester, New York, in 1981, the M.S.E.E. degree from Columbia University, New York, in 1988, an the Ph.D. degree from the University of Colorado, Boulder, in 1993.

From 1993 to 1997, he was Assistant Professor in the School of Electrical and Computer Engineering at the Georgia Institute of Technology. He is currently the Associate Director for the Center on Multidisciplinary Optical Switching Technology (MOST) and Associate Professor in the Department of Electrical and Computer Engineering at the University of California, Santa Barbara. He heads the Optical Communications and Photonic Networks (OCPN) Research. His current research areas are in optical communications, wavelength division multiplexing, photonic packet switched and all-optical networks, all-optical wavelength conversion, optical subcarrier multiplexing, and multispectral optical information processing.

Dr. Blumenthal is recipient of a 1999 Presidential Early Career Award for Scientists and Engineers (PECASE) from the White House and the DoD, a 1994 NSF Young Investigator (NYI) Award and a 1997 Office of Naval Research Young Investigator Program (YIP) Award. He is a member of the Optical Society of America (OSA) and the Lasers and Electro-Optics Society (LEOS).
Jesper Mørk was born in Denmark in 1962. He received the M.Sc. and Ph.D degrees in electrical Engineering from the Technical University of Denmark (TUD), Lyngby, in 1986 and 1988, respectively.

From 1988 to 1996, he was with Tele Danmark Research, since 1994 as a group leader. After half a year with Ericsson in Denmark as Project Manager, he moved to his present position as an Associate Research Professor with Research Center COM, Technical University of Denmark, where he leads the group on modeling of semiconductor devices. His main research interests are ultrafast carrier dynamics in devices, photonic switching, and dynamics in semiconductor lasers. 